\title{
Local Gabor Binary Pattern Whitened PCA: A Novel Approach for Face Recognition from Single Image Per Person
}

\author{
Hieu V. Nguyen, Li Bai, and Linlin Shen \\ School of Computer Science, University of Nottingham, \\ Jubilee Campus, Wollaton Road, Nottingham, NG8 1BB, UK \\ \{vhn, bai\}@cs.nott.ac.uk, llshen@szu.edu.cn \\ http://www.nottingham.ac.uk/cs/
}

\begin{abstract}
One major challenge for face recognition techniques is the difficulty of collecting image samples. More samples usually mean better results but also more effort, time, and thus money. Unfortunately, many current face recognition techniques rely heavily on the large size and representativeness of the training sets, and most methods suffer degraded performance or fail to work if there is only one training sample per person available. This so-called "Single Sample per Person" (SSP) situation is common in face recognition. To resolve this problem, we propose a novel approach based on a combination of Gabor Filter, Local Binary Pattern and Whitened PCA (LGBPWP). The new LGBPWP method has been successfully implemented and evaluated through experiments on 3000+ FERET frontal face images of 1196 subjects. The results show the advantages of our method - it has achieved the best results on the FERET database. The established recognition rates are $98.1 \%, 98.9 \%$, $83.8 \%$ and $81.6 \%$ on the fb, fc, dup I, and dup II probes, respectively, using only one training sample per person.
\end{abstract}

Keywords: Gabor Wavelet, Local Binary Pattern, Whitening, PCA, Feature Selection, Face Recognition.

\section{Introduction}

Face recognition is the identification of individuals from a database of labeled face images. The applications of face recognition can be found in surveillance and security, telecommunication, and smart environments [1]. The biggest challenge of face recognition comes from the numerous variations between images of the same face, such as changes in facial expression, illumination, aging or facial accessories. A robust face recognition system should recognize a face regardless of these intrapersonal facial variations [2].

Discriminating analysis based methods 34 have been widely used to suppress intrapersonal variations. This involves a projection into a subspace whose within-class scatter is small and the between-class scatter is large. A drawback of these methods is that they require large and representative training samples to 
guarantee generalization. However, many face recognition tasks, such as airport security and law enforcement applications, can only offer small sized training samples. Consequently, discriminant analysis tends to over-fit the training data, and fail on future trials using novel (unseen) test data.

Recently, the local binary patterns (LBP) operator has been successfully used for face detection and recognition. LBP encodes both local and global information by a histogram 5. Facial feature extracted by the LBP operator is robust to illumination variations because the LBP features are invariant to monotonic gray-scale changes. However, under varying lighting and aging effects, LBP performance is still not satisfactory [6].

Gabor based face representation is robust to illumination variations and efficient to describe local image features as multi-resolution histograms [78]. The combination of Gabor wavelets with LBP operator has been proposed to represent a face image to obtain robust feature against facial variations [91011. The combined operator is called Local Gabor Binary Patterns (LGBP) operator. Experimental results have demonstrated that the performance of face recognition with LGBP is superior to both of LBP-based approach and Gabor-based approach 91011 .

However, face representation with LGBP is high dimensional due to the multiple Gabor transformations of the LGBP operator [9]. Hence, further dimension reduction is necessary. There are two major categories of methods for dimension reduction: feature selection and feature transformation.

In this paper we introduce a robust face recognition scheme using a single image per person without using discriminant analysis. This scheme has three components: Gabor Filter, Local Binary Pattern (LBP) and Whitened PCA. Gabor and LBP have proven to have very powerful features that complement each other [11] while Standard PCA with whitening can effectively suppress the intrapersonal variation [12. We combine these components in a novel way to solves the SSP problem.

\section{Local Gabor Binary Pattern Whitened PCA Scheme}

\subsection{Face Representation}

The representation approach based on a combination of Gabor Filters and Local Binary Patterns is illustrated in Figure 1] on page 271 [9]. A face image is modeled as a histogram sequence by the following steps:

(1) An input face image is normalized and transformed to obtain multiple Gabor Magnitude Images (GMIs) in frequency domain by applying multi-scale and multi-orientation Gabor filters;

(2) Each GMI is converted to Local Gabor Binary Pattern (LGBP) map;

(3) Each LGBP Map is further divided into non-overlapping rectangle regions with specific size, and histogram is computed for each region; and

(4) The LGBP histograms of all the LGBP Maps are concatenated to form the final histogram sequence as the face representation.

These 4 steps will be described in detail in the following sub-sections. 


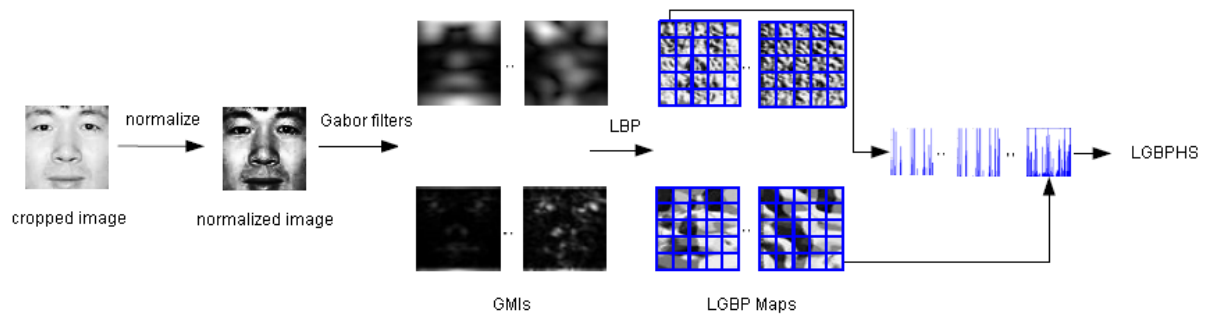

Fig. 1. The proposed LGBPHS face representation approach

Gabor Magnitude Image. Gabor filters most commonly used in face recognition have the form:

$$
\psi_{\mu, \nu}(z)=\frac{\left\|k_{\mu, \nu}\right\|^{2}}{\sigma^{2}} e^{\left(-\left\|k_{\mu, \nu}\right\|^{2}\|z\|^{2} / 2 \sigma^{2}\right)}\left[e^{i k_{\mu, \nu} z}-e^{-\sigma^{2} / 2}\right]
$$

where $\mu$ and $\nu$ define the orientation and scale of the Gabor kernels, $\mathrm{z}=(\mathrm{x}$, y), $\|$.$\| denotes the norm operator, and the wave vector k_{\mu, \nu}=k_{\nu} e^{i \phi_{\mu}}$, where $k_{\nu}=k_{\max } / \lambda^{\nu}$ and $\phi_{\mu}=\pi \mu / 8 . k_{\max }$ is the maximum frequency, $\lambda$ is the spacing factor between filters in the frequency domain.

The Gabor representation of a face image is derived by convolving the face image with the Gabor filters. Let $f(x, y)$ be the face image, its convolution with a Gabor filter $\psi_{\mu, \nu}(z)$ is defined as follows

$$
G_{\psi f}(x, y, \mu, \nu)=f(x, y) * \psi_{\mu, \nu}(z)
$$

where $^{*}$ denotes the convolution operator. Five scales $\nu \in\{0, \cdots, 4\}$ and eight orientations $\mu \in\{0, \cdots, 7\}$ Gabor filters are used. Convolving the image with each of the 40 Gabor filters can then generate the Gabor features. For each Gabor filter, one magnitude value will be computed at each pixel position, which will totally result in 40 Gabor Magnitude Images (GMIs).

Local Gabor Binary Pattern (LGBP). In order to further enhance the information in the Gabor Magnitude Images, we encode the magnitude values with LBP operator. The original LBP operator [13] labels the pixels of an image by thresholding the $3 \times 3$ neighborhood of each pixel $f_{p}(p=0,1, \ldots, 7)$ with the center value $f_{c}$, as shown in Figure 2 on page 272 .

$$
S\left(f_{p}-f_{c}\right)= \begin{cases}1, & f_{p} \geqslant f_{c} \\ 0, & f_{p}<f_{c}\end{cases}
$$

Then, by assigning a binomial factor $2^{p}$ to each $S\left(f_{p}-f_{c}\right)$, the LBP pattern at the pixel is

$$
L B P=\sum_{p=0}^{7} S\left(f_{p}-f_{c}\right) 2^{p}
$$




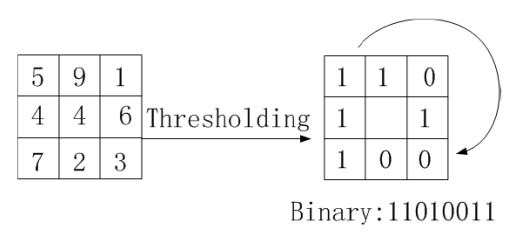

Fig. 2. The basic LBP operator

which characterizes the spatial structure of the local image texture. The operator LGBP denotes the LBP operates on GMI. We denote the transform result at position $(x, y)$ of $(\mu, \nu)$ - GMI as $G_{l g b p}(x, y, \mu, \nu)$, which composes the $(\mu, \nu)$ LGBP Map.

LGBP Histogram Sequence. The histogram $\mathbf{h}$ of an image $f(x, y)$ with gray levels in the range $[0, \mathrm{~L}-1]$ could be defined as

$$
\mathbf{h}_{\mathbf{i}}=\sum_{\mathbf{x}, \mathbf{y}} I\{f(x, y)=i\}, i=0,1, \ldots, L-1
$$

where $i$ is the $\mathrm{i}$-th gray level, $h_{i}$ is the number of pixels in the image with gray level $i$ and

$$
I\{A\}= \begin{cases}1, & \text { Aistrue } \\ 0, & \text { Aisfalse }\end{cases}
$$

Assume each LGBP Map is divided into m regions $R_{o}, R_{1}, \ldots, R_{m-1}$. The histogram of $\mathrm{r}$-th region of the specific LGBP Map (from $(\mu, \nu)$-GMI) is computed by

$$
H_{\mu, \nu, r}=\left(h_{\mu, \nu, r, 0}, h_{\mu, \nu, r, 1}, \ldots, h_{\mu, \nu, r, L-1}\right)
$$

where

$$
h_{\mu, \nu, r, i}=\sum_{(x, y) \in R_{r}} I\left\{G_{l g b p}(x, y, \mu, \nu)=i\right\}
$$

Finally, all the histogram pieces computed from the regions of all the 40 LGBP Map are concatenated to a histogram sequence, $R$, as the final face representation $R=\left(H_{0,0,0}, \ldots, H_{0,0, m-1}, H_{0,1,0}, \ldots, H_{0,1, m-1}, \ldots, H_{4,7, m-1}\right)$.

To solve the high dimensional problem of LGBP representation, we will discuss feature selection and feature transformation methods in the next section.

\subsection{Whitened PCA}

Standard PCA. A classical technique for dimensionality reduction, particularly in face recognition, is principle component analysis (PCA). In order to produce a compact representation, the feature vector is projected into a lower dimensional feature space found by principle components analysis

$$
\mathbf{u}=\mathbf{W}_{\mathbf{p c a}} \mathbf{x}
$$


The input vectors are first transformed by subtracting the mean: $\Phi_{i}=x_{i}-m$. The principal components of the training data set are given by the eigenvectors of its covariance matrix $\sum=\frac{1}{n} \sum_{i=1}^{n} \Phi_{i} \Phi_{i}^{T}$. In practice, only $M(M<n-1)$ eigenvectors having the largest eigenvalues (and, hence, the largest variance in the data set) are kept empirically to form the projection matrix $W_{P C A}$.

PCA technique is optimal in maximizing the scatter of all the projected training samples, meaning PCA maximizes both intrinsic difference and intrapersonal variations in the training set. However, the latter is not useful for classification purposes. This problem is resolved using the LGBPWP scheme with the whitening process.

Whitening Process. PCA has two obvious shortcomings: (1) the leading eigenvectors encode mostly illumination and expression, rather than discriminating information [14]; and (2) Mean-Square-Error (MSE) principle underlying PCA favours low frequencies 151617 and thus loses the discriminating information contained in the high frequency components. The whitening process normalizing the PCA based feature can directly counteract these disadvantages. Specifically, the PCA based feature, $\mathrm{u}$ is subjected to the whitening transformation and yields yet another feature set $\mathrm{w}$ :

$$
\mathrm{w}=\Lambda_{\mathrm{M}}^{-1 / 2} \mathrm{u}
$$

where $\Lambda_{M}^{-1 / 2}=\operatorname{diag}\left\{\lambda_{1}^{-1 / 2}, \lambda_{2}^{-1 / 2}, \ldots, \lambda_{M}^{-1 / 2}\right\}$. The integrated projection matrix $\Lambda_{M}^{-1 / 2} W_{P C A}$ treats variance along all principal component axes as equally significant by weighting components corresponding to smaller eigenvalues more heavily and is arguably appropriate for discrimination. Consequently, the negative influences of the leading eigenvectors are reduced while the discriminating details encoded in trailing eigenvectors are enhanced [12].

\subsection{Feature Selection}

Although LGBP features are very robust, they also cause the 'curse of dimensionality' problem 918] which can be solved by feature selection techniques. Feature selection algorithms such as Adaboost have been very effective 192021. Unfortunately, these techniques cannot be used in this situation because of the SSP assumption, or only one sample per person. Instead, we propose a simple but efficient selection method based on feature variance. Features are ranked based on their variance and highly variant features are selected. These features contain very useful information for classification but they also contain strong intrapersonal variation. This selection method is efficient because when used together with whitened PCA, the intrapersonal variation can be suppressed [12]. Selected features are visualized in Figure 3 on page 274. The gray level represents the number of selected features within each block. As can be expected, the selected features focus on discriminative areas such as eyes, eyebrows, mouth, and nose. 


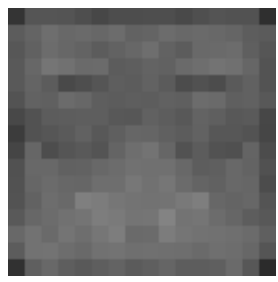

Fig. 3. Distribution of selected features based on their variances

\subsection{Face Recognition}

The original feature vector of the face image is first calculated as detailed in Section 2.1, and the low dimensional LGBPHS-based feature vector, w, is derived using (9). Let $m_{k}^{0}, k=1,2, \ldots, L$, be the prototype, the mean of training samples, for class $\omega_{k}$ in the feature space. The LGBPWP method applies the nearest neighbor (to the mean) rule for classification using similarity measure $\delta$

$$
\delta\left(w, m_{k}^{0}\right)=\min _{j} \delta\left(w, m_{j}^{0}\right) \rightarrow w \in \omega_{k}
$$

The image feature vector, $w$, is classified as belonging to the class of the closest mean, $m_{k}^{0}$, using the similarity measure $\delta$.

Note that when the novel variation, unseen in the training set, is projected onto the feature space, most energy of it will distribute over all the eigenvectors. This is because such variations are somewhat independent of the variance retained by the feature space. In other words, novel variation, projected into the feature space, is incline to evenly affect the projected scale on each component, and thus take more effect on the $L 1$ and $L 2$ distance rather than the vector angle [12]. Therefore, the cosine similarity measure, $\delta_{C S M}$, which is invariant to change in scale, is employed to perform nearest neighbor search in the feature space for face recognition.

$$
\delta_{C S M}\left(w_{1}, w_{2}\right)=\frac{-w_{1}^{T} w_{2}}{\left\|w_{1}\right\| \cdot\left\|w_{2}\right\|}
$$

In the following section we will use experiments on the FERET database to back our claim that the LGBPWP method is robust for face recognition.

\section{$3 \quad$ Experimental Evaluations}

A large scale data set from The FacE REcognition Technology (FERET) database is used to assess the effectiveness and robustness of the new LGBPWP method on the face recognition task. This is a standard test for face recognition technologies [22]. The FERET facial database displays diversity across gender, ethnicity, and age. To obtain a strong and precise assessment of performance, the LGBPWP algorithm is evaluated against different categories of images due to varying lighting, expression, and acquisition time. 


\subsection{Implementation Parameters}

In the preprocessing stage, firstly, face images are all cropped and resized to $128 \times 128$ pixels, and aligned using the locations of two eyes given in FERET database. Then histogram equalization is applied to reduce the illumination effect. Finally, all faces are normalized to zero mean and unit variance.

In the feature extraction stage, the window size of the local region will affect recognition performance. In order to keep more spatial information, in our experiments we empirically choose a small window size of $8 \times 8$ pixels. In order to reduce the dimension of LGBP features, we only use uniform local binary patterns [23]. As a result, the number of LGBP features is $40^{*}(128 / 8) *(128 / 8) * 59=604,160$.

In the feature selection stage, top 25000 variance features, which is much fewer than the original number of features, are selected to feed to Whitened PCA for classification.

All images from the gallery in FERET database are used for PCA training.

\subsection{Variance-Based versus Random Feature Selection}

Intuitively, variance-based feature selection is efficient because highly variant features contain strong discriminant information. However, these features may also contain intrapersonal variation which is not good for recognition. Luckily, whitened PCA tends to suppress the intrapersonal variations. To demonstrate the effectiveness of the variance-based feature selection, we compare it with random feature selection method (LGBPRWP). As the rank curves in Figure 4 on page 275 show, the variance-based method gains much better performance.

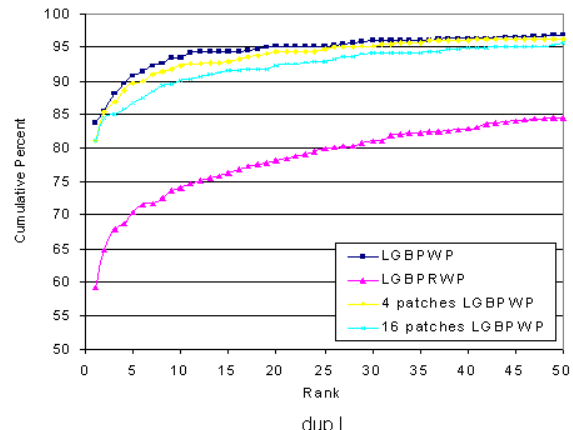

Fig. 4. Rank curves for the dup I (left) and dup II (right) probe sets

\subsection{Holistic PCA versus Ensemble of Piecewise PCAs}

Recently, patch-based and ensemble-based approaches [24] have gained a huge success in terms of recognition performance compared to traditional holistic approaches. However, in the case of LGBPWP, there is no clear difference between patch-based approach and holistic approach. Moreover, the holistic LGBPWP 

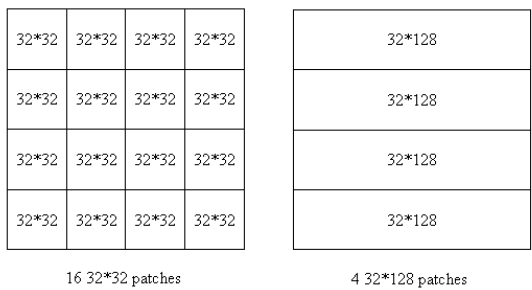

Fig. 5. 16 patches (left) and 4 patches (right) LGBPWP

achieves slightly better results in both dup I and dup II probe sets, as shown in Figure 4 on page 275. Because of the SSP problem, each patch does not contain enough discriminant information to rank matching faces correctly. PCA further compounds the problem. However, patch-based LGBPWP still achieved relatively good performance because of the robustness of Local Gabor Binary Pattern features. To demonstrate, we implemented two patch-based versions of LGBPWP. They are different in terms of patch sizes. As shown in Figure 5] on page 276, the first implementation contains $1632 \times 32$ patches and the second one contains 4 32x128 patches. Rank curves in Figure 4 on page 275 clearly supports our judgement.

\subsection{Results}

In Table 1 on page 276, the rank-1 recognition rates of different algorithms on the FERET probe sets are listed for comparative purpose. As shown in the table, LGBPWP achieves similar results to top algorithms on fb and fc probe sets and a margin about $5 \%$ better than the second best algorithm on dup I and dup II probe sets.

Table 1. Rank-1 recognition rates of different algorithms on the FERET probe sets

\begin{tabular}{c|c|c|c|c}
\hline Methods & fb & fc & dup I & dup II \\
\hline Fisherface & 94.0 & 73.0 & 55.0 & 31.0 \\
\hline Best Results of [25] & 96.0 & 82.0 & 59.0 & 52.0 \\
\hline Results of [23] & 97.0 & 79.0 & 66.0 & 64.0 \\
\hline Direct LGBPHS & 94.0 & 97.0 & 68.0 & 53.0 \\
\hline HGPP & 97.6 & 98.9 & 77.7 & 76.1 \\
\hline GEWC & 96.3 & 99.5 & 78.8 & 77.8 \\
\hline LGBPWP & $\mathbf{9 8 . 1}$ & $\mathbf{9 8 . 9}$ & $\mathbf{8 3 . 8}$ & $\mathbf{8 1 . 6}$ \\
\hline
\end{tabular}

\section{Conclusion and Discussion}

In order to solve the problem of insufficient training data, a new face recognition scheme is proposed that uses single image per person without the need 
for discriminant analysis. The three major components of the proposed method are Gabor Filter, Local Binary Pattern (LBP) and Whitened PCA. The way these three techniques are combined forms a novel approach to solve the SSP problem. Gabor-based features and LBP-based features have proven to be very robust and complementary to each other while standard PCA with whitening can suppress intrapersonal variations very well. Variance-based feature selection is has also proven to be a very efficient when combined with whitened PCA. The results validated the arguments in this paper with the recognition rates of $98.1 \%, 98.9 \%, 83.8 \%$ and $81.6 \%$ on the fb, fc, dup I, and dup II probe using only one training image per person.

Acknowledgments. We acknowledge the support of this research by the University of Nottingham, the UK Engineering and Physical Science Research Council (EPSRC EP/F013477/1), and the China Science Foundation of Shenzhen City (CXQ2008019).

\section{References}

1. Chellappa, R., Wilson, C.L., Sirohey, S., et al.: Human and machine recognition of faces: a survey. Proceedings of the IEEE 83(5), 705-740 (1995)

2. Adini, Y., Moses, Y., Ullman, S.: Face Recognition: The Problem of Compensating for Changes in Illumination Direction. IEEE Transactions on Pattern Analysis and Machine Intelligence, 721-732 (1997)

3. Liu, C., Wechsler, H.: Gabor feature based classification using the enhanced fisher lineardiscriminant model for face recognition. IEEE Transactions on Image Processing 11(4), 467-476 (2002)

4. Swets, D.L., Weng, J.J.: Using Discriminant Eigenfeatures for Image Retrieval. IEEE Transactions on Pattern Analysis and Machine Intelligence, 831-836 (1996)

5. Ahonen, T., Hadid, A., Pietikäinen, M.: Face Description with Local Binary Patterns: Application to Face Recognition. IEEE Transactions on Pattern Analysis and Machine Intelligence, 2037-2041 (2006)

6. Ji, Q.(IEEE Senior Member), Zou, J.(IEEE Member), Nagy, G.(IEEE Fellow): A comparative study of local matching approach for face recognition. IEEE Transactions on Image Processing 16, 2617-2628 (2007)

7. Shan, S., Gao, W., Chang, Y., Cao, B., Yang, P.: Review the strength of Gabor features for face recognition from the angle of its robustness to mis-alignment. In: Proceedings of the 17th International Conference on Pattern Recognition, ICPR 2004, vol. 1 (2004)

8. Wiskott, L., Fellous, J.M., Krüger, N., von der Malsburg, C.: Face Recognition by Elastic Bunch Graph Matching. IEEE Transactions on Pattern Analysis and Machine Intelligence, 775-779 (1997)

9. Zhang, W., Shan, S., Gao, W., Chen, X., Zhang, H.: Local Gabor Binary Pattern Histogram Sequence (LGBPHS): A Novel Non-Statistical Model for Face Representation and Recognition. In: Proc. ICCV, pp. 786-791 (2005)

10. Zhang, W., Shan, S., Chen, X., Gao, W.: Local Gabor Binary Patterns Based On Mutual Information For Face Recognition. International Journal of Image and Graphics 7(4), 777-793 (2007) 
11. Tan, X., Triggs, B., Vision, M.: Fusing Gabor and LBP Feature Sets for KernelBased Face Recognition. In: Zhou, S.K., Zhao, W., Tang, X., Gong, S. (eds.) AMFG 2007. LNCS, vol. 4778, p. 235. Springer, Heidelberg (2007)

12. Deng, W., Hu, J., Guo, J.: Gabor-Eigen-Whiten-Cosine: A Robust Scheme for Face Recognition. In: Zhao, W., Gong, S., Tang, X. (eds.) AMFG 2005. LNCS, vol. 3723, p. 336. Springer, Heidelberg (2005)

13. Ojala, T., Pietikäinen, M., Mäenpää, T.: Multiresolution Gray-Scale and Rotation Invariant Texture Classification with Local Binary Patterns. IEEE Transactions on Pattern Analysis and Machine Intelligence, 971-987 (2002)

14. Etcoff, N., Masoiu, N., Oliyide, O., Pentland, A., Starner, T., Turk, M.: Experiments with eigenfaces. In: Proc. Looking at People Workshop Int'l. Joint Conf. Artifical Intelligence (1993)

15. Sung, K.K., Poggio, T.: Example-based learning for view-based human face detection. IEEE Transactions on Pattern Analysis and Machine Intelligence 20(1), 39-51 (1998)

16. Moghaddam, B., Pentland, A.: Probabilistic visual learning for object representation. IEEE Transactions on Pattern Analysis and Machine Intelligence 19(7), 696-710 (1997)

17. Liu, C.: Gabor-Based Kernel PCA with Fractional Power Polynomial Models for Face Recognition. IEEE Transactions on Pattern Analysis and Machine Intelligence, $572-581$ (2004)

18. Zhang, B., Shan, S., Chen, X., Gao, W.: Histogram of Gabor Phase Patterns (HGPP): A Novel Object Representation Approach for Face Recognition. IEEE Transactions on Image Processing 16(1), 57 (2007)

19. Bai, L., Shen, L.: InfoBoost for Selecting Discriminative Gabor Features. In: Gagalowicz, A., Philips, W. (eds.) CAIP 2005. LNCS, vol. 3691, pp. 423-432. Springer, Heidelberg (2005)

20. Shen, L., Bai, L.: MutualBoost learning for selecting Gabor features for face recognition. Pattern Recognition Letters 27(15), 1758-1767 (2006)

21. Shan, S., Yang, P., Chen, X., Gao, W.: AdaBoost Gabor Fisher Classifier for Face Recognition. In: Zhao, W., Gong, S., Tang, X. (eds.) AMFG 2005. LNCS, vol. 3723, pp. 279-292. Springer, Heidelberg (2005)

22. Phillips, P.J., Wechsler, H., Huang, J., Rauss, P.J.: The FERET database and evaluation procedure for face-recognition algorithms. Image and Vision Computing 16(5), 295-306 (1998)

23. Ahonen, T., Hadid, A., Pietikainen, M.: Face Recognition with Local Binary Patterns. LNCS, pp. 469-481. Springer, Heidelberg (2004)

24. Shan, S., Zhang, W., Su, Y., Chen, X., Gao, W., FRJDL, I.C.T.I., CAS, B.: Ensemble of Piecewise FDA Based on Spatial Histograms of Local (Gabor) Binary Patterns for Face Recognition. In: Proceedings of the 18th international conference on pattern recognition, pp. 606-609 (2006)

25. Phillips, P.J., Moon, H., Rizvi, S.A., Rauss, P.J.: The FERET Evaluation Methodology for Face-Recognition Algorithms. IEEE Transactions on Pattern Analysis and Machine Intelligence, 1090-1104 (2000) 